\title{
Autopercepção negativa da saúde: prevalência e fatores associados entre idosos assistidos em centro de referência
}

\author{
Negative self-perception of health: prevalence and associated \\ factors among elderly assisted in a reference center
}

Jair Almeida Carneiro (https://orcid.org/0000-0002-9501-918X) ${ }^{1}$
Caio Augusto Dias Gomes (https://orcid.org/0000-0003-4860-3276) ${ }^{1}$
Weliton Durães (https://orcid.org/0000-0001-7876-2548) ${ }^{1}$
David Rodrigues de Jesus (https://orcid.org/0000-0003-4792-8300) ${ }^{1}$
Keitlen Lara Leandro Chaves (https://orcid.org/0000-0001-8830-5690) ${ }^{1}$
Cássio de Almeida Lima (https://orcid.org/0000-0002-4261-8226) ${ }^{1}$
Fernanda Marques da Costa (https://orcid.org/0000-0002-3008-7747) ${ }^{2}$
Antônio Prates Caldeira (https://orcid.org/0000-0002-9990-9083) ${ }^{1}$
${ }^{1}$ Universidade Estadual de Montes Claros. Av. Prof. Rui Braga s/n, Vila Mauricéia. 39401-089 Montes Claros MG Brasil. cassioenf2014@gmail.com ${ }^{2}$ Centro Universitário FIPMoc. Montes Claros MG Brasil.

\begin{abstract}
The negative self-perception of health is a relevant construct for the analysis of the elderly population health conditions and should be recognized as a guiding tool for health promotion actions. We aimed to verify the prevalence and the associated factors to a negative self-perception of health by elderly assisted in a reference center. This is an analytical cross-sectional study with convenience sampling conducted in 2015. The negative self-perception association with health-related sociodemographic variables was investigated. Bivariate analysis followed by multiple analysis by Poisson regression was performed to verify the variables associated with the outcome. Three hundred sixty elderly were evaluated. The negative self-perception of health prevalence was $60.5 \%$. The following associated factors were identified in the final model: age range 65-79 years $(P R=1 ; \quad C I 95 \%=0.648-0.974 ; \quad p=0.027)$; frailty $(P R=1.28 ; C 195 \%=1.07-1.54 ; p=0.007)$; depressive symptoms ( $P R=1.40 ; C 195 \%=1.19$ 1.67; $p=0.000)$; and providing care to someone $(P R=1.49 ; \quad C I 95 \%=1.18-1.88 ; p=0.001)$. The elevated prevalence of negative self-perception of health and the associated factors point to the need for effective health promotion actions and more specific care for the elderly assisted in the reference center.
\end{abstract}

Key words Self-assessment, Elderly, Elderly health, Aging, Health services for the elderly
Resumo A autopercepção do estado de saúde constitui relevante construto para a análise das condiçães de saúde da população idosa e deve ser reconhecida como instrumento norteador de ações de promoção da saúde. Objetivou-se verificar a prevalência e os fatores associados à autopercepção negativa da saúde em idosos assistidos em serviço de referência. Pesquisa transversal analítica, realizada em 2015, com amostra por conveniência. Investigou-se a associação entre autopercepção negativa da saúde e variáveis sociodemográficas e relacionadas à saúde. Para averiguar as variáveis associadas ao desfecho, processaram-se análises bivariadas, seguidas de análise múltipla por Regressão de Poisson. Foram avaliados 360 idosos. A prevalência de autopercepção negativa da saúde foi de 60,5\%. No modelo final, identificaram-se os fatores associados: idade na faixa de 65 a 79 anos $(R P=1$; IC95\%=0,648-0,974; $p=0,027)$; fragilidade ( $R P=1,28$; IC95\% $=1,07$ 1,54; $p=0,007)$; sintomas depressivos $(R P=1,40$; IC95\%=1,19-1,67; $p=0,000)$; prestar cuidados a alguém ( $R P=1,49 ;$ IC95\%=1,18-1,88; $p=0,001)$. A elevada prevalência de autopercepção negativa da saúde e os fatores associados indicam a necessidade de ações efetivas de promoção da saúde e cuidados mais específicos aos idosos assistidos no centro de referência.

Palavras-chave Autoavaliação, Idoso, Saúde do idoso, Envelhecimento, Serviços de saúde para idosos 


\section{Introdução}

A autopercepção do estado de saúde é um indicador subjetivo da percepção do indivíduo sobre a própria saúde, confiável e passível de ser aplicado de maneira eficaz, rápida e de baixo custo na avaliação da saúde de grupos populacionais ${ }^{1,2}$. Incorpora tanto componentes físicos, cognitivos e emocionais, como aspectos relacionados ao bemestar e à satisfação com a própria vida ${ }^{3-5}$. Assim, tem sido largamente evidenciado como importante aspecto do bem-estar individual e coletivo ${ }^{6}$, sendo recomendado para análises da saúde das populações ${ }^{3}$.

A investigação da autopercepção de saúde tem sido utilizada amplamente em estudos populacionais, e o interesse sobre o assunto também tem crescido em pesquisas abrangendo os idosos $^{1,4,6}$. Seja no cenário nacional ${ }^{1,3,6-11}$ ou internacional ${ }^{12-17}$, verifica-se um declínio no estado geral de saúde desse grupo, afetando a autopercepção. Assim, essa medida tem a capacidade de predizer, de maneira robusta e consistente, a morbimortalidade, o declínio da capacidade funcional, a inatividade e a depressão. Então, trata-se de um relevante construto para a análise das condições de saúde da população idosa, que deve ser reconhecido como instrumento útil, com consequente aplicação em ações de promoção da saúde desse grupo ${ }^{1-4,6,18}$.

A verificação da autopercepção da saúde entre idosos apresenta expressiva relevância mundial $^{2,7-9,12-18}$. Em estudos internacionais, observa-se que tal desfecho tem relação com aspectos como: nível de educação superior ${ }^{17}$, ter emprego atual ${ }^{17}$, viuvez ${ }^{16}$, suporte social ${ }^{13}$, doença crôni$\mathrm{ca}^{12,13,16}$, depressão $\mathrm{o}^{12,14,16}$, capacidade funcional ${ }^{12}$, atividades instrumentais de vida diária ${ }^{14}$, atividade física ${ }^{15,17}$, medo de cair ${ }^{16}$, síndrome da fragilidade $^{16}$, obesidade ${ }^{17}$, satisfação com serviços de saúde ${ }^{12}$ e mortalidade ${ }^{12}$. E o binômio autopercepção de saúde-qualidade de vida é um consenso nessas pesquisas ${ }^{11-17}$.

Esse assunto também é importante no Brasil, que, nos últimos anos, apresenta acelerado processo de envelhecimento populacional, com impactos e desafios para os serviços de saúde. É plausível inferir que eventos frequentes entre idosos, como maior número de doenças crônicas não transmissíveis e incapacidade funcional, repercutam em uma percepção negativa da própria saúde. E, como se refere a uma avaliação subjetiva, a autopercepção da saúde possui caráter multidimensional, envolve estilos de vida, além de aspectos psicológicos, demográficos e socioeco- nômicos ${ }^{2,7-9,18,19}$. A heterogeneidade de fatores associados indica que a saúde dos idosos se vincula a determinantes que se aproximam do conceito ampliado de saúde 3 .

Ainda há vários aspectos da saúde subjetiva desse segmento etário dos brasileiros por investigar. O conhecimento dos aspectos envolvidos na percepção da saúde pode revelar os subgrupos mais vulneráveis de idosos, bem como ancorar os serviços de saúde nas iniciativas de promoção da saúde e de uma melhor qualidade de vida ${ }^{6}$. Em função do crescimento da população idosa e da aplicabilidade da autopercepção do estado de saúde como indicativo das condições gerais de saúde da população, pesquisas sobre essa temática são necessárias. Isso porque possibilitarão esclarecer, acompanhar e comparar resultados, a fim de orientar a tomada de decisão no tocante à formulação de políticas e intervenções de saúde mais apropriadas às especificidades dos idosos ${ }^{3}$.

As singularidades que abarcam a autopercepção de saúde justificam a condução de estudos locais e regionais, que possam subsidiar os gestores do setor saúde na elaboração de medidas para melhorar as condições de saúde da comunidade, especialmente a idosa, mais crescente nos últimos anos ${ }^{5,9}$. Desse modo, elucidar os fatores que influenciam na autopercepção de saúde dos idosos poderá contribuir para identificar quais dimensões precisam ser melhor ponderadas e reconhecidas pelos profissionais de saúde ${ }^{3}$.E, embora tal percepção constitua um importante indicador para a vigilância da saúde geral do idoso, ainda é pouco abordada na literatura brasileira ${ }^{10}$. Ressalta-se que há lacunas de evidências especialmente em regiões carentes e distantes dos grandes centros metropolitanos, devido à escassez de estudos epidemiológicos nesses locais, a exemplo do Norte do estado de Minas Gerais (MG), Brasil.

Na referida região, destaca-se o "Centro Mais Vida de Referência em Assistência à Saúde do Idoso (CRASI)". Como o Brasil vivencia um acelerado processo de envelhecimento populacional, com crescentes demandas para o sistema de saú$\mathrm{de}^{19}$, a Secretaria de Estado da Saúde de Minas Gerais implantou o Programa Mais Vida e a Rede de Atenção à Saúde do Idoso do Estado de Minas Gerais. O CRASI é um dos serviços dessa rede, conta com equipe multiprofissional e contrarreferência para as unidades básicas de saúde, sendo referência para os 96 municípios da região $0^{20}$. Entretanto, tal serviço, de indubitável potencial para a atenção à saúde da população idosa, é um ponto ainda recente da citada rede, se desconhecem pesquisas sobre o perfil e a autopercepção 
da saúde dos usuários assistidos nesse local. Essas singularidades demonstram a necessidade de investigações de caráter multidimensional, nesse cenário, que poderão ser utilizadas no estabelecimento de estratégias visando o envelhecimento saudável. Logo, justifica-se a realização da presente pesquisa, considerando a conjuntura abordada, a necessidade de se agregar novas informações epidemiológicas sobre a temática, o contexto socioeconômico e cultural do local investigado e o pioneirismo devido ao cenário serviço de referência da atenção secundária.

Este estudo teve por objetivo verificar a prevalência e os fatores associados à autopercepção negativa da saúde em idosos assistidos em serviço de referência.

\section{Métodos}

Trata-se de uma pesquisa transversal e analítica, com abordagem quantitativa. Foi realizada com idosos usuários do "CRASI Eny Faria de Oliveira”, o único serviço de referência específico para a clientela idosa na região, prestando atendimento multidimensional por meio de uma equipe multiprofissional composta por assistente social, enfermeiro, farmacêutico, fisioterapeuta, fonoaudiólogo, médico geriatra, nutricionista, psicólogo e terapeuta ocupacional. Está situado na cidade de Montes Claros, Norte do estado de MG - Brasil. A cidade conta com uma população de 402.027 habitantes e representa o principal polo urbano regional.

A amostra foi obtida por amostragem de conveniência conforme a demanda atendida, durante o período de maio a julho de 2015. A média de atendimentos por equipe multiprofissional era de aproximadamente 1.450 por mês, durante o período de coleta dos dados. Foi adotado como critério de inclusão: ter idade igual ou superior a 65 anos, pois o instrumento aplicado para mensurar a fragilidade, Escala de Fragilidade de Edmonton (EFE), foi validado apenas para pessoas nessa faixa etária ${ }^{21}$. Por sua vez, foram excluídos idosos com incapacidade cognitiva, segundo a avaliação da família; déficit auditivo não corrigido que impedisse o entendimento das perguntas; bem como recusa à participação na pesquisa por parte do idoso ou de seus familiares. Os participantes eram residentes de Montes Claros e dos demais municípios da região coberta pelo referido serviço.

Os entrevistadores foram previamente treinados. Os dados foram coletados de forma primária por meio de contato direto e entrevistas, tendo os idosos como respondentes. O instrumento de coleta de dados utilizado teve como base trabalhos similares, de base populacional ${ }^{1,3,4,6,7,9,10,22}$, foi previamente testado em estudo piloto, sendo composto pelas variáveis apresentadas a seguir.

A variável dependente foi a autopercepção de saúde, apurada por meio da seguinte interrogação feita ao idoso: "Como o(a) Senhor(a) classificaria seu estado de saúde?” As opções de resposta eram: "Muito bom", “Bom”, "Regular”, "Ruim" ou "Muito ruim". Para análise, as respostas foram dicotomizadas e assumiu-se como percepção positiva da saúde as respostas "Muito bom" e "Bom", e percepção negativa da saúde o somatório das respostas "Regular", "Ruim" e "Muito ruim", seguindo investigações prévias sobre o tema ${ }^{2,8-10,23}$.

As variáveis independentes foram agrupadas em sociodemográficas e relativas à saúde, conforme descrito a seguir:

- Variáveis sociodemográficas: sexo (masculino $\mathrm{x}$ feminino); idade (65-79 anos $\mathrm{x} \geq 80$ anos); cor da pele autorreferida (branca $\mathrm{x}$ outras); escolaridade, em anos de estudo (até quatro anos $\mathrm{x}>$ quatro anos); situação conjugal (casado ou união estável x sem companheiro); arranjo familiar (não reside sozinho $\mathrm{x}$ reside sozinho); condição de prestar cuidados a alguém (não x sim); renda própria (não x sim); renda familiar mensal (até um salário mínimo $\mathrm{x}>$ um salário mínimo). Todas essas variáveis foram averiguadas mediante o autorrelato dos participantes.

- Variáveis relativas à saúde: fragilidade (não frágil $\mathrm{x}$ frágil); presença de comorbidades crônicas - todas segundo autorrelato (hipertensão arterial, diabetes mellitus, incontinência urinária, doença osteoarticular, doença cardíaca, acidente vascular cerebral - categorizadas em não x sim); polifarmácia (não x sim) - considerado o uso de cinco ou mais medicamentos ${ }^{20}$; internação no último ano (não x sim); sintomas depressivos (não $\mathrm{x} \operatorname{sim}$ ); quedas (não $\mathrm{x} \operatorname{sim}$ ). Todas essas variáveis foram investigadas considerando o autorrelato dos participantes. A fragilidade e os sintomas depressivos foram avaliados por instrumentos específicos.

Para aferir a fragilidade, aplicou-se a EFE, adaptada culturalmente e validada para a língua portuguesa $^{21}$. Trata-se de um instrumento que avalia nove domínios: cognição, estado de saúde, independência funcional, suporte social, uso de medicação, nutrição, humor, continência urinária e desempenho funcional, distribuídos em 11 itens com pontuação de 0 a 17 . A pontuação da EFE pode variar entre $0-4$, indica que não há pre- 
sença de fragilidade; 5-6, aparentemente vulnerável para fragilidade; 7-8, fragilidade leve; 9-10, fragilidade moderada; e 11 ou mais, fragilidade severa. No presente estudo, para a análise dos dados, os resultados da variável foram dicotomizados em dois níveis: não frágil (escore final $\leq 6$ ) e frágil (escore $>6)^{21,24}$.

Os sintomas depressivos foram determinados por meio da Escala Geriátrica de Depressão, que possui 15 questões. Tal instrumento também já foi validado nacionalmente, é composto por perguntas negativas/afirmativas. $\mathrm{O}$ resultado de seis ou mais pontos indica sintomatologia depressiva; dessa forma, o ponto de corte adotado neste trabalho foi 5/6 (não/sim - equivalente a não caso/ (aso $)^{25}$.

Realizou-se análise bivariada, seguida de análise múltipla por Regressão de Poisson, com variância robusta. Em cada uma das análises, foram calculadas as Razões de Prevalência (RP) e foram considerados com os seus respectivos intervalos de confiança a 95\%. Para a análise múltipla, avaliaram-se apenas as variáveis que mostraram associação até o nível de 20\% (p < 0,20), na análise bivariada. No modelo final, após a análise múltipla, foram mantidas apenas as variáveis que se mostraram associadas até o nível de 5\% (p < $0,05)$. As análises foram realizadas com uso do programa Statistical Package for the Social Sciences (SPSS), versão 17.0 (SPSS for Windows, Chicago, EUA).

O projeto de pesquisa foi aprovado pelo Comitê de Ética em Pesquisa da Universidade Estadual de Montes Claros (Unimontes). Todos os participantes foram orientados sobre a pesquisa e apresentaram sua anuência, através da assinatura de Termo de Consentimento Livre e Esclarecido.

\section{Resultados}

Participaram do estudo 360 idosos com idade igual ou superior a 65 anos. A faixa etária predominante foi entre 65 e 79 anos, que representou $75,3 \%$ da população em estudo. A média de idade do grupo foi de 75 anos (DP $\pm 7,6)$. A maioria era do sexo feminino $(78,0 \%)$, não residia sozinha $(83,0 \%)$, tinha cor da pele "outras" $(62,5 \%)$, afirmou ter renda própria $(97,5 \%)$ e possuía até quatro anos de estudo $(85,8 \%)$.

O registro de internação hospitalar, com permanência superior a 24 horas, foi apontado por $21,0 \%$. Aspectos de morbidade autorreferida investigados revelaram que 76,9\% eram hipertensos, $54,4 \%$ sofreram queda no último ano, $43,9 \%$ referiram doenças osteoarticulares, 37,2\% apresentaram sintomas depressivos, 21,9\% possuíam doença cardíaca, 20,3\% eram diabéticos e 10,6\% tinham histórico de acidente vascular cerebral.

Quanto à autopercepção da saúde, 142 $(39,4 \%)$ idosos apresentaram uma percepção positiva de sua própria saúde ("muito boa" ou "boa"); 143 (39,7\%) descreveram sua saúde como "regular"; enquanto $75(20,8 \%)$ referiram-se à própria saúde como "ruim" ou "muito ruim”. Considerando como percepção negativa as categorias "regular", "ruim" e "muito ruim", a prevalência de autopercepção negativa da saúde neste estudo foi de $60,5 \%$. Porém, ao se incluir somente as categorias "ruim" e "muito ruim", essa prevalência foi de 20,8\%.

As análises bivariadas entre autopercepção de saúde negativa e demais variáveis são demonstradas nas Tabelas 1 e 2.

No modelo final, os fatores associados à autopercepção negativa da saúde foram: idade na faixa de 65 a 79 anos (RP = 1; IC95\% = 0,648$0,974 ; \mathrm{p}=0,027)$; fragilidade $(\mathrm{RP}=1,28$; IC95\% $=1,07-1,54 ; \mathrm{p}=0,007) ;$ sintomas depressivos $(\mathrm{RP}$ $=1,40$; IC95\% = 1,19-1,67; $\mathrm{p}=<0,001)$; e prestar cuidados a alguém $(\mathrm{RP}=1,49$; IC95\% $=1,18$ $1,88 ; \mathrm{p}=0,001)($ Tabela 3$)$.

\section{Discussão}

O presente estudo, ao considerar as categorias "regular", "ruim" e "muito ruim", evidenciou elevada prevalência do desfecho autopercepção negativa do estado de saúde em idosos assistidos pelo CRASI, e permitiu conhecer os fatores associados.

Os valores de prevalências desse desfecho oscilam na literatura, registrando-se os achados: variou de 12,6 a 51,9\% em revisão sistemática sobre a autopercepção da saúde em idosos brasileiros ${ }^{3} ; 57,5 \%$ em município de grande porte de $\mathrm{MG}^{9} ; 51,2 \%$ em Florianópolis-SC ${ }^{10} ; 49,6 \%$ em pesquisa realizada em três cidades brasileiras $^{7} ; 10,9 \%$ em Campinas-SP ${ }^{6}$. Em um centro de referência de Belo Horizonte-MG a prevalência foi superior: $70,1 \%{ }^{26}$. Em outros países, igualmente se observam diversos achados: na Alemanha $62,5 \%$ dos idosos relataram autopercepção negativa do estado de saúde ${ }^{14}$; em Xangai, China, houve o valor de $56,8 \%{ }^{12}$; enquanto em Santiago de Cali, Colômbia, a prevalência foi de $40,1 \%{ }^{16}$.

Observa-se que a prevalência de autopercepção negativa de saúde difere de forma considerável entre os trabalhos, o que exige cautela na 
Tabela 1. Resultado da análise bivariada entre autopercepção negativa da saúde e variáveis sociodemográficas em idosos assistidos no Centro Mais Vida de Referência em Assistência à Saúde do Idoso, Montes Claros, Minas Gerais, Brasil, $2015(\mathrm{n}=360)$.

\begin{tabular}{|c|c|c|c|c|c|c|c|}
\hline \multirow{3}{*}{ Variáveis Independentes } & \multicolumn{4}{|c|}{ Autopercepção Negativa da Saúde } & \multirow[t]{3}{*}{$\mathbf{R P}$} & \multirow[t]{3}{*}{ IC 95\% } & \multirow[t]{3}{*}{$\mathbf{P}$} \\
\hline & \multicolumn{2}{|c|}{ Sim } & \multicolumn{2}{|c|}{ Não } & & & \\
\hline & $\mathbf{N}$ & $\%$ & $\mathbf{N}$ & $\%$ & & & \\
\hline \multicolumn{8}{|l|}{ Sexo } \\
\hline Masculino & 45 & 57,0 & 34 & 43,0 & 1 & & \\
\hline Feminino & 173 & 61,6 & 108 & 38,4 & 1,08 & $0,87-1,33$ & 0,45 \\
\hline \multicolumn{8}{|l|}{ Faixa etária } \\
\hline $65-79$ anos & 170 & 62,7 & 101 & 37,3 & 1 & & \\
\hline$\geq 80$ anos & 48 & 53,9 & 41 & 46,1 & 0,86 & $0,69-1,06$ & 0,14 \\
\hline \multicolumn{8}{|l|}{ Cor da pele autorreferida } \\
\hline Branca & 92 & 68,1 & 43 & 31,9 & 1 & & \\
\hline Outras & 126 & 56,0 & 99 & 44,0 & 0,82 & $0,69-0,86$ & 0,02 \\
\hline \multicolumn{8}{|l|}{ Escolaridade em anos de estudo } \\
\hline Até 4 anos & 190 & 61,5 & 119 & 38,5 & 1 & & \\
\hline$>4$ anos & 28 & 54,9 & 23 & 45,1 & 1,12 & $0,86-1,45$ & 0,37 \\
\hline \multicolumn{8}{|l|}{ Situação conjugal } \\
\hline Com companheiro & 87 & 59,6 & 59 & 40,4 & 1 & & \\
\hline Sem companheiro & 131 & 61,2 & 83 & 38,8 & 1,02 & $0,86-1,21$ & 0,75 \\
\hline \multicolumn{8}{|l|}{ Arranjo familiar } \\
\hline Não reside sozinho & 177 & 59,2 & 122 & 40,8 & 1 & & \\
\hline Reside sozinho & 41 & 67,2 & 20 & 32,8 & 1,13 & $0,93-1,38$ & 0,24 \\
\hline \multicolumn{8}{|l|}{ Presta cuidados a alguém } \\
\hline Não & 178 & 67,2 & 87 & 32,8 & 1 & & \\
\hline Sim & 40 & 42,1 & 55 & 57,9 & 0,62 & $0,48-0,80$ & 0,00 \\
\hline \multicolumn{8}{|l|}{ Renda própria } \\
\hline Não & 5 & 55,6 & 4 & 44,4 & 1 & & \\
\hline Sim & 213 & 60,7 & 138 & 39,3 & 0,91 & $050-1,65$ & 0,75 \\
\hline \multicolumn{8}{|l|}{ Renda familiar mensal } \\
\hline Até 1 salário mínimo & 74 & 64,3 & 41 & 35,7 & 1 & & \\
\hline$>1$ salário mínimo & 144 & 58,8 & 101 & 41,2 & 1,09 & $0,92-1,30$ & 0,31 \\
\hline
\end{tabular}

comparação dos resultados. Embora as perguntas sobre essa variável, assim como as opções de resposta, sejam similares entre as investigações, os achados não são unânimes. Tem-se como provável explicação o fato de que a forma de categorização não é consensual entre as pesquisas. Essas discrepâncias também podem ser devido a flutuações de curto prazo na saúde ou doença, ocasionadas por variações cíclicas relacionadas ao bem-estar. Ademais, a autopercepção de saúde tem relação com várias dimensões essenciais da saúde do idoso. E podem existir diferenças entre as regiões estudadas, quanto aos aspectos socioeconômicos, demográficos e relativos ao sistema local de saúde, os quais podem interferir no estado de saúde da população idosa e na prevalência do desfecho analisado ${ }^{10}$.
Convém comentar que essas diferenças também podem ter implicações inclusive no cenário desta investigação, que atende usuários de diversos municípios, com realidades e perfis assistenciais bem distintos entre si. Entretanto, tais divergências não significam uma menos valia do indicador, que representa uma medida bastante útil em estudos epidemiológicos, e que reflete vários aspectos do perfil de saúde, dos cuidados e da utilização dos serviços de saúde ${ }^{2,9}$.

Apesar de tais discrepâncias, os resultados relativos à autopercepção da saúde pelos idosos participantes deste estudo não foram satisfatórios. É esperado que, com o avançar da idade e as consequentes alterações fisiológicas e sociais na vida do indivíduo, ocorra uma piora no estado de saúde e, por conseguinte, na autoavaliação desse 
Tabela 2. Resultado da análise bivariada entre autopercepção negativa da saúde e variáveis relativas à saúde em idosos assistidos no Centro Mais Vida de Referência em Assistência à Saúde do Idoso, Montes Claros, Minas Gerais, Brasil, $2015(\mathrm{n}=360)$.

\begin{tabular}{|c|c|c|c|c|c|c|c|}
\hline \multirow{3}{*}{ Variáveis Independentes } & \multicolumn{4}{|c|}{ Autopercepção Negativa da Saúde } & \multirow[t]{3}{*}{$\mathbf{R P}$} & \multirow[t]{3}{*}{ IC 95\% } & \multirow[t]{3}{*}{$\mathbf{P}$} \\
\hline & \multicolumn{2}{|c|}{ Sim } & \multicolumn{2}{|c|}{ Não } & & & \\
\hline & $\mathbf{N}$ & $\%$ & $\mathbf{N}$ & $\%$ & & & \\
\hline \multicolumn{8}{|l|}{ Fragilidade } \\
\hline Não Frágil & 95 & 50,0 & 95 & 50,0 & 1 & & \\
\hline Frágil & 123 & 72,4 & 47 & 27,6 & 1,44 & $1,22-1,75$ & 0,00 \\
\hline \multicolumn{8}{|l|}{ Incontinência urinária } \\
\hline Não & 152 & 55,7 & 121 & 44,3 & 1 & & \\
\hline Sim & 66 & 75,9 & 21 & 24,1 & 1,36 & $1,16-1,59$ & 0,00 \\
\hline \multicolumn{8}{|l|}{ Hipertensão Arterial } \\
\hline Não & 56 & 61,5 & 35 & 38,5 & 1 & & \\
\hline $\operatorname{Sim}$ & 162 & 60,2 & 107 & 39,8 & 0,97 & $0,81-1,18$ & 0,82 \\
\hline \multicolumn{8}{|l|}{ Diabetes Mellitus } \\
\hline Não & 177 & 61,7 & 110 & 38,3 & 1 & & \\
\hline $\operatorname{Sim}$ & 41 & 56,2 & 32 & 43,8 & 0,91 & $0,72-1,13$ & 0,39 \\
\hline \multicolumn{8}{|l|}{ Doença Cardíaca } \\
\hline Não & 168 & 59,8 & 113 & 40,2 & 1 & & \\
\hline $\operatorname{Sim}$ & 50 & 63,3 & 29 & 36,7 & 1,05 & $0,87-1,28$ & 0,57 \\
\hline \multicolumn{8}{|l|}{ Doença Osteoarticular } \\
\hline Não & 114 & 56,4 & 88 & 43,6 & 1 & & \\
\hline $\operatorname{Sim}$ & 104 & 65,8 & 54 & 34,2 & 1,16 & $0,98-1,37$ & 0,07 \\
\hline \multicolumn{8}{|l|}{ Acidente Vascular Cerebral } \\
\hline Não & 189 & 58,7 & 133 & 41,3 & 1 & & \\
\hline $\operatorname{Sim}$ & 29 & 76,3 & 9 & 23,7 & 1,30 & $1,06-1,58$ & 0,03 \\
\hline \multicolumn{8}{|l|}{ Polifarmácia } \\
\hline Não & 138 & 57,5 & 102 & 42,5 & 1 & & \\
\hline $\operatorname{Sim}$ & 80 & 66,7 & 40 & 33,3 & 1,15 & $0,98-1,37$ & 0,09 \\
\hline \multicolumn{8}{|l|}{ Internação no último ano } \\
\hline Não & 180 & 63,4 & 104 & 36,6 & 1 & & \\
\hline Sim & 38 & 50,0 & 38 & 50,0 & 0,78 & $0,62-1,00$ & 0,05 \\
\hline \multicolumn{8}{|l|}{ Sintomas depressivos } \\
\hline Não & 112 & 49,6 & 114 & 50,4 & 1 & & \\
\hline Sim & 106 & 79,1 & 28 & 20,9 & 1,59 & $1,36-1,86$ & 0,00 \\
\hline \multicolumn{8}{|l|}{ Quedas } \\
\hline Não & 112 & 68,3 & 52 & 31,7 & 1 & & \\
\hline Sim & 106 & 54,1 & 90 & 45,9 & 0,79 & $0,67-0,93$ & 0,00 \\
\hline
\end{tabular}

estado. Contudo, essa situação não deve ser vista como algo natural e não pode ser negligenciada. A alta prevalência de autopercepção negativa da saúde em idosos requer atenção especial, principalmente por parte dos profissionais da Atenção Primária à Saúde (APS) e dos serviços de referência, devido ao seu impacto direto e indireto na piora da saúde ${ }^{27}$.

Logo, são necessários esforços, no sentido de garantir o acesso a uma atenção à saúde de qualidade, com acompanhamento criterioso dos profissionais de saúde, atividades de lazer e promoção da saúde, bem como maior integração social. Isso também aumentaria a disposição desse segmento populacional em aderir aos protocolos terapêuticos e comportamentos de saúde que minimizem os riscos de uma evolução negativa do processo de envelhecimento ${ }^{8}$, recomendações estas também indicadas na literatura internacio$\mathrm{nal}^{12,15,17,28,29}$.

No que concerne aos fatores associados, este estudo evidenciou maior prevalência de autoper- 
Tabela 3. Fatores associados à autopercepção negativa da saúde em idosos assistidos no Centro Mais Vida de Referência em Assistência à Saúde do Idoso, Montes Claros, Minas Gerais, Brasil, 2015 ( $\mathrm{n}=360)$.

\begin{tabular}{lrrr}
\hline $\begin{array}{c}\text { Variáveis } \\
\text { independentes }\end{array}$ & $\begin{array}{c}\text { RP } \\
\text { ajustada }\end{array}$ & IC95\% & $\begin{array}{c}\mathbf{p} \\
\text { valor }\end{array}$ \\
\hline Faixa etária & & & \\
$\quad 65-79$ anos & 1 & & \\
$\geq 80$ anos & 0,79 & $0,648-0,974$ & 0,027 \\
Fragilidade & & & \\
$\quad$ Não Frágil & 1 & & \\
$\quad$ Frágil & 1,28 & $1,07-1,54$ & 0,007 \\
Sintomas depressivos & & & \\
$\quad$ Não & 1 & & \\
$\quad$ Sim & 1,40 & $1,19-1,67$ & $<0,001$ \\
$\begin{array}{l}\text { Presta cuidados a } \\
\text { alguém }\end{array}$ & & & \\
$\quad$ Não & 1 & & \\
Sim & 1,49 & $1,18-1,88$ & 0,001 \\
\hline
\end{tabular}

cepção negativa da saúde entre idosos entre 65 a 79 anos, ao passo que, entre os indivíduos em idade mais avançada, essa percepção foi melhor, achado este também identificado em pesquisas realizadas na Espanha ${ }^{30}$ e em Belo Horizonte- $\mathrm{MG}^{23}$. Esses resultados sugerem um processo de adaptação ou aceitação dos idosos longevos em relação às condições de saúde consideradas próprias do envelhecimento, bem como de comorbidades, incapacidades e hospitalizações que podem lhes afetar ${ }^{30}$, conforme corrobora uma pesquisa realizada na República da Letônia, Europa $^{28}$. Há uma redução nas expectativas com relação à saúde de indivíduos nas últimas fases da vida, pois o fato de terem sobrevivido até aquele estágio já representa um estado de saúde positivo. Também é possível que tal resultado tenha sofrido o efeito da sobrevivência seletiva, que decorre de apenas os indivíduos mais sadios terem sobrevivido até as faixas etárias mais avançadas e, por isso, possuírem melhores percepções sobre sua saúde $\mathrm{s}^{4,30}$.

Todavia, outras investigações, no Brasil ${ }^{4,31}$, em Xangai $^{12}$ e em municípios espanhóis ${ }^{13}$ indicaram que o aumento da idade esteve associado à autoavaliação ruim do estado de saúde. Sabe-se que, quanto mais a idade atinge faixas etárias longevas, mais o estado geral de saúde tende a deteriorar. Em adição, a maioria das doenças crônicas é mais comum em idades mais avançadas, prejudicando substancialmente o desfecho em questão ${ }^{4,30}$.
Os resultados desta pesquisa mostraram associação entre autopercepção negativa da saúde e o fato de possuir fragilidade, o que também foi constatado em pesquisa realizada em município de grande porte do estado de $\mathrm{MG}^{9}$ e em outra na cidade de Santiago de Cali, Colômbia ${ }^{16}$. A fragilidade é definida como um estado clinicamente reconhecível de aumento da vulnerabilidade, resultante do declínio na reserva e na função em múltiplos sistemas fisiológicos associado ao envelhecimento, comprometendo a capacidade de lidar com condições estressoras. É determinada pelo efeito combinado do envelhecimento biológico com condições crônicas, aumenta a susceptibilidade às doenças e influencia a capacidade funcional, além de prejudicar a dependência, mobilidade, estabilidade postural e lucidez do idoso ${ }^{32-34}$. Portanto, é esperado que qualquer grau de fragilidade esteja associado a uma percepção negativa da saúde ${ }^{9,22}$.

A princípio, esperava-se maior frequência da fragilidade no cenário desta investigação, já que o idoso frágil tem indicação absoluta para uma avaliação especializada, multidimensional e multidisciplinar, conforme os critérios de referenciamento para o CRASI ${ }^{20}$. Ressalta-se que a associação identificada demonstra o impacto negativo da condição de fragilidade na vida do indivíduo, o que requer maior zelo dos profissionais de saúde e intervenções para a prevenção de quadros como esse $\mathrm{e}^{9,22,29}$.

$\mathrm{O}$ atual trabalho evidenciou outro importante achado: associação da variável desfecho com sintomas depressivos, resultado também registrado na literatura nacional ${ }^{1,3,8,26,27,35,36}$, bem como nos citados estudos realizados em Xangai ${ }^{12}$, na Alemanha ${ }^{14}$, em Santiago de Cali ${ }^{16}$ e na República da Letônia ${ }^{28}$. Salienta-se que a própria avaliação ruim sobre a própria saúde pode ser vista como um sintoma depressivo ${ }^{36}$. As condições associadas ao surgimento de sintomas depressivos podem, concomitantemente, influenciar a autopercepção negativa da saúde pelo idoso. Tais sintomas representam impacto negativo em todos os aspectos na vida do idoso, afetando também a sua vida em família e na comunidade. Ademais, estão associados a desfechos adversos, como comprometimento da saúde física e maior utilização de serviços $^{27,35,36}$.

A associação constatada é um resultado consistente: os sintomas em questão constituem um dos problemas mais frequentes no campo da geriatria. Apesar da subjetividade desse aspecto, a forma como o indivíduo se vê em relação ao seu estado geral se vincula intimamente com os qua- 
dros depressivos. A pior saúde orgânica também aumenta a exposição a esses quadros, sendo que esse aumento é diretamente relacionado ao número de doenças crônicas, que foi elevado nesta pesquisa. Sendo assim, configura-se uma situação que demanda cuidados redobrados por parte da família, dos cuidadores e profissionais de saúde, devido à sua repercussão na piora da saúde do idoso. Recomenda-se a identificação precoce de casos e melhor abordagem nos serviços de APS e de referência, a fim de proporcionar diagnósticos e intervenções oportunas e precisas, além de diminuir os custos para o sistema de saúde ${ }^{27}$.

A necessidade de maior suporte aos idosos se faz pertinente, ponderando que os participantes desta pesquisa que prestam cuidados a outra pessoa também apresentaram pior autopercepção do seu estado de saúde. Atualmente, com o aumento da longevidade, há tendência de aumento no número de cuidadores idosos, então é crescente o número de idosos sendo cuidados por outros idosos. Sendo que os idosos mais jovens apresentam mais energia para cuidar dos indivíduos mais velhos ${ }^{37}$.

Possivelmente, essa também é uma realidade entre os participantes desta investigação. Todavia, a tarefa de cuidar é complexa, por isso as atividades realizadas diariamente pelo cuidador idoso a algum indivíduo dependente pode gerar sobrecarga, bem como desgaste da saúde física e menta ${ }^{38,39}$. Logo, acredita-se que privilegiar a avaliação da sobrecarga dos idosos cuidadores, e incluí-los em planos de cuidados com ações específicas de apoio, pode auxiliar tanto o cuidador como quem é cuidado por este ${ }^{38}$.

Por fim, informa-se que o presente trabalho apresenta limitações. Ele é derivado de um estudo transversal, não sendo possível concluir a existência de associação causal entre a autopercepção negativa da saúde e os fatores associados, o que seria factível em desenhos longitudinais. As variáveis foram aferidas por informação do próprio idoso, e, embora esse seja um procedimento válido e utilizado em diversos estudos, pode ter sido comprometido pela memória e pela baixa escolaridade. Além disso, tratou-se de amostra de conveniência, circunscrita a um centro de referência, em que a validade externa fica limitada e os resultados podem ser extrapolados apenas para população e cenário semelhantes.

A despeito dessas limitações, esta pesquisa teve amostra suficiente para o ajuste dos modelos de regressão aos principais fatores de confusão de interesse. Possui caráter inédito, posto que o cenário foi um centro de referência, enquanto os demais trabalhos são em sua maioria de base populacional; os resultados e as associações registradas foram corroborados pela literatura. E, à luz do conhecimento gerado neste trabalho, profissionais e gestores podem planejar medidas promotoras de melhorias na atenção à saúde do idoso, mais apropriadas ao contexto de vida e saúde desse grupo.

\section{Conclusão}

Este estudo evidenciou elevada prevalência de autopercepção negativa do estado de saúde entre os idosos assistidos em um centro de referência. Identificou-se que estar na faixa de 65 a 79 anos, apresentar fragilidade, possuir sintomas depressivos e prestar cuidados a alguém se mantiveram como fatores associados a essa prevalência. Esses achados sinalizam a necessidade de ações efetivas de promoção da saúde e cuidados mais específi$\cos$, direcionados principalmente às necessidades daqueles idosos que autoavaliaram negativamente o seu estado de saúde e se enquadraram nos referidos fatores. 


\section{Colaboradores}

JA Carneiro: concepção do estudo, do planejamento e da coleta de dados, da análise e interpretação dos dados, da redação do artigo. CAD Gomes, W Durães, DR Jesus e KLL Chaves: concepção do estudo, da coleta de dados, da interpretação dos dados, da redação do artigo. CA Lima: concepção do estudo, do planejamento e da coleta de dados, da interpretação dos dados, da redação e revisão crítica do artigo. FM Costa: concepção do estudo, do planejamento e da coleta de dados, da análise e interpretação dos dados, da revisão crítica do artigo. AP Caldeira: orientou todas as etapas do estudo e da elaboração do artigo, participou da concepção do estudo, do planejamento da coleta de dados, da análise e interpretação dos dados, da revisão crítica do artigo. Todos os autores leram e aprovaram a versão final do artigo.

\section{Agradecimentos}

À Universidade Estadual de Montes Claros (Unimontes) e à Fundação de Amparo à Pesquisa do Estado de Minas Gerais (FAPEMIG), por bolsas do Programa Institucional de Bolsas de Iniciação Científica (PIBIC).

\section{Referências}

1. Lima-Costa MF, Firmo JOA, Uchôa E. The structure of self-rated health among older adults: the Bambuí health and ageing study (BHAS). Rev Saude Publica 2004; 38(6):827-834.

2. Aguilar-Palacio I, Carrera-Lasfuentes P, Rabanaque MJ. Salud percibida y nivel educativo en España: tendencias por comunidades autónomas y sexo (20012012). Gaceta Sanitaria 2015; 29(1):37-43.

3. Pagotto V, Bachion MM, Silveira EA. Autoavaliação da saúde por idosos brasileiros: revisão sistemática da literatura. Rev Panam Salud Publica 2013; 33(4):302310.

4. Pavão ALB, Werneck GL, Campos MR. Autoavaliação do estado de saúde e a associação com fatores sociodemográficos, hábitos de vida e morbidade na população: um inquérito nacional. Cad Saude Publica 2013; 29(4):723-734.

5. Melo ACF, Nakatani AYK, Pereira LV, Menezes RL, Pagotto V. Prevalência de doenças musculoesqueléticas autorreferidas segundo variáveis demográficas e de saúde: estudo transversal de idosos de Goiânia/GO. Cad Saude Colet 2017; 25(2):138-143.

6. Borim FSA, Barros MBA, Neri AL. Autoavaliação da saúde em idosos: pesquisa de base populacional no Município de Campinas, São Paulo, Brasil. Cad Saude Publica 2012; 28(4):769-780.

7. Silva RJS, Smith-Menezes A, Tribess S, Rómo-Perez V, Virtuoso Júnior JS. Prevalência e fatores associados à percepção negativa da saúde em pessoas idosas no Brasil. Rev Bras Epidemiol 2012; 15(1):49-62.

8. Loyola Filho AI, Firmo JOA, Uchôa E, Lima-Costa MF. Fatores associados à autoavaliação negativa da saúde entre idosos hipertensos e/ou diabéticos: resultados do projeto Bambuí. Rev Bras Epidemiol 2013; 16(3):559-571.

9. Medeiros SM, Silva LSR, Carneiro JA, Ramos GCF, Barbosa ATF, Caldeira AP. Factors associated with negative self-rated health among non-institutionalized elderly in Montes Claros, Brazil. Cien Saude Colet 2016; 21(11):3377-3386

10. Confortin SC, Giehl MWC, Antes DL, Schneider IJC, d'Orsi E. Positive self-rated health in the elderly: a population-based study in the South of Brazil. Cad Saude Publica 2015; 31(5):1049-1060.

11. Höfelmann DA, Garcia LP, Freitas LRS. Self-rated health in Brazilian adults and elderly: Data from the $\mathrm{Na}$ tional Household Sample Survey 2008. Salud Publica Mex 2014; 56(6):603-611.

12. Chen Y, While AE, Hicks A. Self-rated health and associated factors among older people living alone in Shanghai. Geriatr Gerontol Int 2015; 15(4):457-464.

13. Lorenzo T, Millán-Calenti JC, Lorenzo-López L, Sánchez A, Maseda A. Predictores de mala salud autopercibida en una población de personas mayores. Rev Esp Geriatr Gerontol 2013; 48(6):272-275.

14. Nützel A, Dahlhaus A, Fuchs A, Gensichen J, König HH, Riedel-Heller S, Maier W, Schäfer I, Schön G, Weyerer S, Wiese B, Scherer M, van den Bussche H, Bickel H. Self-rated health in multimorbid older general practice patients: a cross-sectional study in Germany. BMC Fam Pract 2014; 15(1):1-12. 
15. Rosenkranz RR, Duncan MJ, Rosenkranz SK, Kolt GS. Active lifestyles related to excellent self-rated health and quality of life: cross sectional findings from 194,545 participants in The 45 and Up Study. BMC Public Health 2013; 13(1071):1-12.

16. Ocampo-Chaparro JM, Zapata-Ossa HJ, CubidesMunévar AM, Curcio CL, Villegas JD, Reyes-Ortiz CA. Prevalence of poor self-rated health and associated risk factors among older adults in Cali, Colombia. Colomb Med 2013; 44(4):224-231.

17. Khalaila R. Socioeconomic status, health behaviors, obesity and self-rated health among older arabs in Israel. J Cross Cult Gerontol 2017; 32(1):115-130.

18. Carvalho AT, Malta DC, Barros MBA, Oliveira PNFP, Mendonça DMMV, Barros H. Desigualdades na autoavaliação de saúde: uma análise para populações do Brasil e de Portugal.Cad Saude Publica 2015; 31(11):2449-2461.

19. Veras R. Envelhecimento populacional contemporâneo: demandas, desafios e inovações.Rev Saude Publica 2009; 43(3):548-554.

20. Brasil. Minas Gerais. Secretaria do Estado de Saúde (SES). Resolução SES no 2.603, de 07 de dezembro de 2010. Dispõe sobre o Programa Mais Vida - Rede de Atenção à Saúde do Idoso de Minas Gerais, e dá outras providências. Belo Horizonte: SES; 2010.

21. Fabrício-Wehbe SCC, Schiaveto FV, Vendrusculo TRP, Haas VJ, Dantas RAS, Rodrigues RAP. Cross-cultural adaptation and validity of the "Edmonton Frail Scale-EFS" in a brazilian elderly sample. Rev Latino Am Enfermagem 2009; 17(6):1043-1049.

22. Carneiro JA, Cardoso RR, Durães MS, Guedes MCA, Santos FL, Costa FM, Caldeira AP. Frailty in the elderly: prevalence and associated factors. Rev Bras Enferm 2017; 70(4):747-752.

23. Jardim R, Barreto SM, Giatti L. Self-reporting and secondary informant reporting in health assessments among elderly people.Rev Saude Publica 2010; 44(6):1120-1129.

24. Rolfson DB, Majumdar SR, Tsuyuki RT, Tahir A, Rockwood K. Validity and reliability of the Edmonton Frail Scale. Age Ageing 2006; 35(1):526-529.

25. Almeida OP, Almeida SA. Confiabilidade da versão brasileira da Escala de Depressão em Geriatria (GDS) versão reduzida. Arq Neuro Psiquiatr 1999; 57(2B):421-426.

26. Ribeiro EG, Matozinhos FP, Guimarães GL, Couto AM, Azevedo RS, Mendoza IYQ. Self-perceived health and clinical-functional vulnerability of the elderly in Belo Horizonte/Minas Gerais. Rev Bras Enferm 2018; 71(Supl. 2):860-867.

27. Nogueira EL, Rubin LL, Giacobbo SS, Gomes I, Cataldo NA. Screening for depressive symptoms in older adults in the Family Health Strategy, Porto Alegre, Brazil. Rev Saude Publica 2014; 48(3):368-377.

28. Löfqvist C, Tomsone S, Iwarsson S, Horstmann V, Haak M. Changes inhome and health over nine years among very old people in Latvia - results from the ENABLE-AGE Project. J Cross Cult Gerontol 2017; 32(1):17-29.
29. Kanwar A, Singh M, Lennon R, Ghanta K, McNallan SM, Roger VL. Frailty and health-related quality of life among residents of long-term care facilities. J Aging Health 2013; 25(5):792-802.

30. Damian J, Ruigomez A, Pastor V, Martin-Moreno JM. Determinants of self assessed health among Spanish older people living at home. J Epidemiol Community Health 1999; 53(7):412-416.

31. Sousa AI, Silver LD. Perfil sociodemográfico e estado de saúde auto-referido entre idosas de uma localidade de baixa renda. Esc Anna Nery Rev Enferm 2008; 12(4):706-716.

32. Xue QL. The frailty syndrome: definition and natural history. Clin Geriatr Med 2011; 27(1):1-15.

33. Lacas A, Rockwood K. Frailty in primary care: a review of its conceptualization and implications for practice. BMC Med 2012; 10(1):10-14.

34. Clegg A, Young J, Iliffe S, Rikkert MO, Rockwood K. Frailty in elderly people. Lancet 2013; 381 (2):752-762.

35. Ramos GCF, Carneiro JA, Barbosa ATF, Mendonça JMG, Caldeira AP. Prevalência de sintomas depressivos e fatores associados em idosos no norte de Minas Gerais: um estudo de base populacional. J Bras Psiquiatr 2015; 64(2):122-131.

36. Borges LJ, Benedetti TRB, Xavier AJ, d'Orsi E. Fatores associados aos sintomas depressivos em idosos: estudo Epi Floripa. Rev Saude Publica 2013; 47(4):701-710.

37. Orlandi AAS, Brito TRP, Ottaviani AC, Rossetti ES, Zazzetta MS, Gratão ACM, Orlandi FS, Pavarini SCI. Profile of older adults caring for other older adults in contexts of high social vulnerability. Esc Anna Nery 2017; 21(1):1-8.

38. Fuhrmann AC, Bierhals CCBK, Santos NO, Paskulin LMG. Association between the functional capacity of dependant elderly people and the burden of family caregivers. Rev Gaúcha Enferm 2015; 36(1):14-20.

39. Morais D, Terassi M, Inouye K, Luchesi BM, Pavarini SCI. Chronic pain in elderly caregivers at different levels of frailty. Rev Gaúcha Enferm 2016; 37(4):1-7.

Artigo apresentado em 10/12/2017

Aprovado em 06/07/2018

Versão final apresentada em 08/07/2018 